\title{
Hemoglobin A1c Levels Affect Visit-to-visit Variability of Lipid Profiles in Patients Undergoing Elective Percutaneous Coronary Intervention: A Retrospective Study
}

\section{Duanbin Li}

Zhejiang University School of Medicine Sir Run Run Shaw Hospital

\section{Cao Wang}

Zhejiang University School of Medicine Sir Run Run Shaw Hospital

\section{Liding Zhao}

Zhejiang University School of Medicine Sir Run Run Shaw Hospital

\section{Yi Luan}

Zhejiang University School of Medicine Sir Run Run Shaw Hospital

\section{Ya Li}

Zhejiang University School of Medicine Sir Run Run Shaw Hospital

\section{Guosheng Fu}

Zhejiang University School of Medicine Sir Run Run Shaw Hospital

\section{Zhaoyang Chen}

Xiehe Affiliated Hospital of Fujian Medical University

Wenbin Zhang ( $\sim$ 3313011@zju.edu.cn )

Department of Cardiology, Sir Run Run Shaw Hospital, School of Medicine Zhejiang University, 3 East Qingchun Road, Hangzhou, Zhejiang Province, People's Republic of China. https://orcid.org/0000-00034746-9570

\section{Research}

Keywords: Hemoglobin A1c, HbA1c, Variability, Lipid profiles, Percutaneous coronary intervention

Posted Date: November 5th, 2020

DOl: https://doi.org/10.21203/rs.3.rs-101772/v1

License: (c) (i) This work is licensed under a Creative Commons Attribution 4.0 International License. Read Full License 


\section{Abstract}

\section{Background}

Both hemoglobin A1c (HbA1c) levels and visit-to-visit variability of lipid profiles are risk factors for cardiovascular disease (CVD). We conducted a retrospective cohort study to explore the relationship between $\mathrm{HbA} 1 \mathrm{c}$ and lipid variability.

\section{Methods}

We retrospectively collected baseline and follow-up data on patients who underwent elective percutaneous coronary intervention (PCl) from January 2009 to April 2019. Univariate and multivariate linear regression analyses were performed to assess the association between $\mathrm{HbA} 1 \mathrm{c}$ and lipid variability. Subgroup analyses employed multivariate linear regression analyses.

Results

A total of 4,445 patients were enrolled in the study. The median age and value of $\mathrm{HbA} 1 \mathrm{c}$ were $64 \mathrm{yrs}$ old and $5.9 \%$, respectively, $64 \%$ had hypertension and $25.5 \%$ diabetes. The variability of low-density lipoprotein cholesterol (LDL-C), non-high-density lipoprotein cholesterol (non-HDL-C), total cholesterol (TC), and triglyceride (TG) was each significantly higher in patients with $\mathrm{HbA} 1 \mathrm{c} \geq 6.5 \%$ than those with $\mathrm{HbA1c} ₫ 6.5 \%$ group. Multivariate linear regression indicated that $\mathrm{HbA1c}$ level was a potential risk factor for the variability of LDL-C, non-HDL-C, TC and TG, which was independent of the mean values of lipids. Subgroup analyses demonstrated that the relationship between $\mathrm{HbA} 1 \mathrm{c}$ and the variability of $\mathrm{LDL}-\mathrm{C}$, nonHDL-C, TC, and TG did not importantly vary across several subgroups. These results remained consistent when lipid variability was represented by the standard deviation (SD), coefficient of variation (CV) and variability independent of the mean (VIM), respectively.

Conclusion

$\mathrm{HbA1c}$ is a potential risk factor for the variability of LDL-C, non-HDL-C, TC and TG in patients undergoing elective PCl.

\section{Background}

Cardiovascular disease (CVD) remains the leading cause of death globally and is one of the most common complications of diabetes mellitus (DM) [1, 2]. Hemoglobin A1c ( HbA1c) is usually regarded as an indicator representing the average blood glucose levels over the past 2-3 months and is often employed to assess glycemic control[3]. Several epidemiological studies[4, 5] have demonstrated associations between elevated HbA1c levels and adverse CVD outcomes in patients with type $2 \mathrm{DM}$. Moreover, even within the normal reference range, increased blood glucose levels are associated with an increased risk of CVD[6]. 
Traditionally, mean values of indicators or exposures are often employed to estimate the prognostic risk for CVD. For example, higher low-density lipoprotein cholesterol (LDL-C) levels and lower high-density lipoprotein cholesterol (HDL-C) levels are associated with an increased risk of CVD[7, 8]. However, if there is wide variability in the exposure, the mean value will no longer be reliable. In recent years, more attention is being focused on the variability of these exposures. With particular emphasis on lipid profiles, the visitto-visit variability of LDL-C, HDL-C, non-high-density lipoprotein cholesterol (non-HDL-C), total cholesterol (TC) and triglyceride (TG) has each been demonstrated to be a potential risk factor for CVD[9-13]; these associations are independent of the mean levels of the exposures and traditional cardiovascular risk factors. Based on intravascular ultrasound examination, elevated variability of LDL-C has been shown to independently promote the progression of atherosclerosis, which is the crucial underlying pathology of $\mathrm{CVD}^{[14]}$. In general, lipid variability, an independent risk factor for CVD, is attracting considerable attention.

Both $\mathrm{HbA} 1 \mathrm{c}$ and the variability of blood lipids are risk factors for CVD. However, whether there is any relationship between $\mathrm{HbA} 1 \mathrm{c}$ and the variability of lipids is still unclear. Therefore, the purpose of this study is to investigate the relationship between $\mathrm{HbA} 1 \mathrm{c}$ and lipid variability in patients undergoing elective percutaneous coronary intervention (PCl).

\section{Methods}

\section{Study subjects}

The study population comprised all consecutive patients who attended the Sir Run Run Shaw Hospital, Zhejiang University in China, from January 2009 to April 2019. Eligibility criteria for inclusion into the study were: (1) patients who underwent elective $\mathrm{PCl}$, (2) sufficient clinical information such as $\mathrm{HbA1c}$ and lipid profiles were available at baseline and follow-ups, (3) patients were followed up at 1, 3, 6, 9, and 12 months following $\mathrm{PCl}$ according to the prescribed follow-up procedures. Patients with acute myocardial infarction (MI), active cardiopulmonary diseases, heart failure, severe liver and/or renal insufficiency, cancer, acute or chronic infection and other serious diseases were excluded from the study. A total of 4,445 patients were finally enrolled in the study. All PCl procedures were performed by experienced interventional cardiologists using the recommended guidelines[15]. Baseline and follow-up measurements of exposures were ascertained from fasting venous blood samples (at least $8 \mathrm{hrs}$ overnight). Levels of HbA1c, LDL-C, HDL-C, non-HDL-C, TC and TG were determined at each follow-up. Standard follow-up procedures and examinations were employed for all patients who had undergone elective PCI. The Ethics Committee of Sir Run Run Shaw Hospital of Zhejiang University approved the study (20200803-34).

\section{Assessment of variability in lipid profiles}

Baseline and follow-up values of lipids were measured by a blood chemistry analyzer (Hitachi 747; Hitachi, Tokyo, Japan). The variability of lipids reflected the degree of fluctuation of individual blood lipid levels during the 1-year follow-up period. To assess the lipid variability more comprehensively, the 
following 3 indicators were used:(1) standard deviation (SD); (2) coefficient of variation (CV): The CV of blood lipids was defined as follows, $C V=(S D / m e a n) \times 100(\%)$; and (3) variability independent of the mean (VIM): VIM was calculated as $\left(\mathrm{SD} / \mathrm{mean}^{\beta}\right) \times 100(\%)$, in which $\beta$ was derived from the fitting of the curve and was the regression coefficient based on the natural logarithm of SD and the natural logarithm of the mean[16].

\section{Definitions}

The level of $\mathrm{HbA1} \mathrm{c}$ was defined as the mean value during a 1-year follow-up period and was calculated using values of $\mathrm{HbA} 1 \mathrm{c}$ measured at follow-up. Diabetes mellitus was defined as a fasting serum glucose $\geq 126 \mathrm{mg} / \mathrm{dL}$, a history of diabetes or the current use of antidiabetic medications. Hypertension was defined as blood pressure $\geq 140 / 90 \mathrm{mmHg}$, a documented history of hypertension or on antihypertensive medications. Regular statin therapy was defined as atorvastatin $\leq 20 \mathrm{mg}$ or rosuvastatin $\leq 10 \mathrm{mg}$ per day. Intensive statin therapy was defined as atorvastatin $\geq 40 \mathrm{mg}$ or rosuvastatin $\geq 20 \mathrm{mg}$ per day. Particular attention was paid to patients' compliance with statin use. Heart failure was defined by ejection fraction $(\mathrm{EF})<40 \%$ or N-terminal pro B-type natriuretic peptide (NT-pro $\mathrm{BNP})>2000 \mathrm{pg} / \mathrm{ml}$ without renal failure. Body mass index (BMI) was calculated as weight in kilograms, divided by height in meters squared. Glomerular filtration rate (GFR) was estimated using the Japanese Society of Nephrology equation as follows: estimated GFR (eGFR) $\left(\mathrm{mL} / \mathrm{min} / 1.73 \mathrm{~m}^{2}\right)=194 \times$ serum creatinine ${ }^{-1.094} \times$ age $^{-0.287}(\times 0.739$ for women $)[17]$.

\section{Statistical analysis}

Continuous variables were presented as median (interquartile range) or mean (standard deviation). Categorical variables were represented as frequency (\%). Baseline characteristics were compared between patients with $\mathrm{HbA} 1 \mathrm{c} \varangle 6.5 \%$ and $\mathrm{HbA} 1 \mathrm{c} \geq 6.5 \%$ using chi-square tests for categorical variables and nonparametric tests for continuous variables. Univariate and multivariate linear regression analyses were used to evaluate the relationship between $\mathrm{HbA} 1 \mathrm{c}$ and lipid variability. Subgroup analyses employed multivariate linear regression analyses. A value of $P<0.05$ ( 2 sided) was considered statistically significant. Statistical analysis was performed using SPSS software version 22.0 (SPSS Inc., Chicago, IL, USA).

\section{Result}

\section{Patient characteristics}

The 4,445 patients who had undergone elective $\mathrm{PCl}$ at the Sir Run Run Shaw Hospital between January 2009 and April 2019 comprised 3,538 males and 907 females. Overall, the median age of subjects was 64 (58-70) years old, with a median BMI of $24.73(22.77-26.18) \mathrm{kg} \cdot \mathrm{m}^{-2}, 64 \%$ had hypertension and $25.5 \%$ had type 2 diabetes mellitus. The median value of $\mathrm{HbA} 1 \mathrm{c}$ was $5.9 \%$, with the interquartile range from $5.5 \%$ to $6.7 \%$ for all subjects. The variability of LDL-C, non-HDL-C, TC and TG was each significantly higher in patients with $\mathrm{HbA} 1 \mathrm{c} \geq 6.5 \%$ than in those with $\mathrm{HbA} 1 \mathrm{c} \otimes 6.5 \%$. Patients with $\mathrm{HbA} 1 \mathrm{c} \geq 6.5 \%$ 
appeared to have higher BMI (24.73[23.38-26.67] vs. 24.62[22.48-26.00]); $\mathrm{p}<0.001)$, more likely to have hypertension $(70.0 \%$ vs. $62.5 \%$; $<<0.001)$ than those with $\mathrm{HbA1c} \otimes 6.5 \%$. Table 1 summarizes the baseline characteristics of the enrolled patients categorized by HbA1c levels.

Table 1 Baseline characteristics of patients 


\begin{tabular}{|c|c|c|c|c|}
\hline Characteristics & $\begin{array}{l}\text { Total } \\
(\mathrm{n}=4445)\end{array}$ & 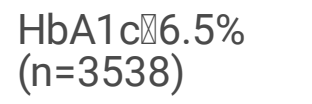 & $\begin{array}{l}\mathrm{HbA} 1 \mathrm{c} \geq 6.5 \% \\
(\mathrm{n}=907)\end{array}$ & $\begin{array}{l}\mathrm{P} \text { - } \\
\text { value }\end{array}$ \\
\hline \multicolumn{5}{|l|}{ Demographic information } \\
\hline Age (Years) & $\begin{array}{l}64.00(58.00- \\
70.00)\end{array}$ & $\begin{array}{l}64.00(58.00- \\
70.00)\end{array}$ & $\begin{array}{l}64.00(58.00- \\
71.00)\end{array}$ & 0.158 \\
\hline Male, N (\%) & $3187(71.7)$ & $2549(72.0)$ & 638(70.3) & 0.391 \\
\hline $\mathrm{BMI},\left(\mathrm{kg} / \mathrm{m}^{2}\right)$ & $\begin{array}{l}24.73(22.77- \\
26.18)\end{array}$ & $\begin{array}{l}24.62(22.48- \\
26.00)\end{array}$ & $\begin{array}{l}24.73(23.38- \\
26.67)\end{array}$ & $\begin{array}{l}\square \\
0.001\end{array}$ \\
\hline Current smoking, N (\%) & 1102(24.8) & $890(25.2)$ & $212(23.4)$ & 0.388 \\
\hline Hypertension, N (\%) & $2845(64.0)$ & $2210(62.5)$ & $635(70.0)$ & $\begin{array}{l}\square \\
0.001\end{array}$ \\
\hline Type 2 diabetes, N (\%) & $1133(25.5)$ & $327(9.2)$ & $806(88.9)$ & $\begin{array}{l}\square \\
0.001\end{array}$ \\
\hline Previous MI, N (\%) & 164(3.7) & $129(3.6)$ & $35(3.9)$ & 0.786 \\
\hline Previous PCl, N (\%) & $342(7.7)$ & $278(7.9)$ & $64(7.1)$ & 0.581 \\
\hline LDL-C<1.8 mmol/L, N (\%) & $3729(83.9)$ & $2984(84.3)$ & $745(82.1)$ & 0.127 \\
\hline \multicolumn{5}{|l|}{ Laboratory examination } \\
\hline $\mathrm{HbA1c}(\%)$ & $5.9(5.50-6.70)$ & $5.7(5.40-6.00)$ & 7.6(6.90-8.70) & $\begin{array}{l}\square \\
0.001\end{array}$ \\
\hline LDL-C (mean, mmol/L) & $1.78(1.49-2.13)$ & $1.78(1.50-2.12)$ & $1.77(1.47-2.18)$ & 0.834 \\
\hline HDL-C (mean, mmol/L) & $1.02(0.88-1.20)$ & $1.05(0.90-1.22)$ & $0.97(0.83-1.14)$ & $\begin{array}{l}\square \\
0.001\end{array}$ \\
\hline non-HDL-C (mean, mmol/L) & $2.46(2.10-2.90)$ & $2.47(2.10-2.87)$ & $2.44(2.08-2.95)$ & 0.806 \\
\hline TC (mean, mmol/L) & $3.66(3.21-4.16)$ & $3.67(3.25-4.12)$ & $3.62(3.15-4.23)$ & 0.219 \\
\hline TG (mean, mmol/L) & $1.36(1.04-1.82)$ & $1.35(1.04-1.78)$ & $1.38(1.05-1.95)$ & 0.051 \\
\hline LDL-C (SD) & $0.47(0.27-0.75)$ & $0.45(0.25-0.74)$ & $0.50(0.29-0.76)$ & 0.014 \\
\hline HDL-C (SD) & $0.13(0.08-0.18)$ & $0.13(0.08-0.18)$ & $0.13(0.08-0.18)$ & 0.577 \\
\hline non-HDL-C (SD) & $0.43(0.25-0.70)$ & $0.42(0.25-0.70)$ & $0.47(0.27-0.73)$ & 0.004 \\
\hline $\mathrm{TC}(\mathrm{SD})$ & $0.59(0.35-0.93)$ & $0.56(0.33-0.90)$ & $0.66(0.39-1.00)$ & $\begin{array}{l}\square \\
0.001\end{array}$ \\
\hline \multirow[t]{2}{*}{ TG (SD) } & $0.33(0.19-0.55)$ & $0.32(0.19-0.52)$ & $0.37(0.20-0.65)$ & $\begin{array}{l}\square \\
0.001\end{array}$ \\
\hline & $89.25(75.22-$ & 89.22(75.96- & 89.19(70.65- & 0.848 \\
\hline
\end{tabular}




\begin{tabular}{|lllll|}
\hline \multicolumn{1}{|c}{ eGFR $\left(\mathrm{mL} / \mathrm{min} / 1.73 \mathrm{~m}^{2}\right)$} & $98.11)$ & $97.70)$ & $99.01)$ & \\
\hline Baseline medication & & & & \\
\hline ACEI, N (\%) & $1102(24.8)$ & $876(24.8)$ & $226(24.9)$ & 0.897 \\
\hline ARB, N (\%) & $1565(35.2)$ & $1180(33.4)$ & $385(42.4)$ & 0 \\
\hline Beta blocker, N (\%) & $2614(58.8)$ & $2092(59.1)$ & $522(57.6)$ & 0.429 \\
\hline CCB, N (\%) & $1325(29.8)$ & $1020(28.8)$ & $305(33.6)$ & 0.007 \\
\hline Aspirin, N (\%) & $4294(96.6)$ & $3424(96.8)$ & $870(95.9)$ & 0.244 \\
\hline Clopidogrel, N (\%) & $3592(80.8)$ & $2889(81.7)$ & $703(77.5)$ & 0.007 \\
\hline Ticagrelor, N (\%) & $805(18.1)$ & $613(17.3)$ & $192(21.2)$ & 0.008 \\
\hline Statin, N (\%) & $4401(99.0)$ & $3507(99.1)$ & $894(98.6)$ & 0.151 \\
\hline Intensive statin, N (\%) & $845(19.0)$ & $660(18.7)$ & $185(20.3)$ & 0.064 \\
\hline $\begin{array}{l}\text { Statin combined with ezetimibe, } \\
\text { N (\%) }\end{array}$ & $956(21.5)$ & $764(21.6)$ & $192(21.2)$ & 0.818 \\
\hline
\end{tabular}

Data are expressed as median (25\%-75\%) or $\mathrm{n}(\%)$. Total indicates total group; SD, standard deviation; $\mathrm{BMI}$, body mass index; MI, myocardial infarction; $\mathrm{LDL}-\mathrm{C}<1.8 \mathrm{mmol} / \mathrm{L}, \mathrm{LDL}-\mathrm{C}<1.8 \mathrm{mmol} / \mathrm{L}$ at the firstmonth follow-up after percutaneous coronary intervention (PCI); LDL-C, low-density lipoprotein cholesterol; HDL-C, high-density lipoprotein cholesterol; TC, total cholesterol; TG, triglyceride; eGFR, estimated glomerular filtration rate; $\mathrm{ACEl}$, angiotensin-converting enzyme inhibitors; $\mathrm{ARB}$, angiotensin receptor blocker; CCB, calcium channel blocker

\section{Results of univariate and multivariate linear regression for the variability of lipid profiles}

Based on clinical considerations and differences in baseline characteristics according to $\mathrm{HbA1C}$ categories, the following factors were included in the univariate analysis: $\mathrm{HbA} 1 \mathrm{c}$, age, gender, BMI, current smoking, hypertension, eGFR, types of statin, intensive statin treatment, statin combined with ezetimibe and the corresponding mean values of lipids. Factors that were significant in the univariate analyses $(p<0.05)$ were included in multivariate linear regression analysis.

Multivariate analysis for the effect of $\mathrm{HbA} 1 \mathrm{c}$ on lipid variability was conducted in all patients and demonstrated that $\mathrm{HbA} 1 \mathrm{c}$ was a potential risk factor for the variability of lipids, which included LDL-C (regression coefficients of HbA1c on SD [ $\beta]$ 0.014, standard error [SE] 0.004, P-value冈0.001), non-HDL-C

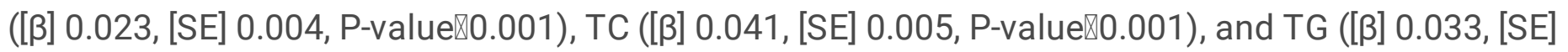
0.005 , P-value $\mathbb{0} 0.001)$. Besides, HbA1c remained a potential risk factor for the variability of LDL-C, non- 
HDL-C, TC and TG when CV and VIM were employed to represent lipid variability, respectively (see Table 2 to 5).

Table 2 Results of univariate and multivariate linear regression for the variability of LDL-C

\begin{tabular}{|c|c|c|c|c|c|c|c|c|c|c|c|c|c|c|c|c|c|c|}
\hline & \multicolumn{6}{|c|}{$\mathrm{SD}$} & \multicolumn{6}{|c|}{$\mathrm{cV}$} & \multicolumn{6}{|c|}{ VIM } \\
\hline & \multicolumn{3}{|c|}{ Univariate regression } & \multicolumn{3}{|c|}{ Multiple regression } & \multicolumn{3}{|c|}{ Univariate regression } & \multicolumn{3}{|c|}{ Multiple regression } & \multicolumn{3}{|c|}{ Univariate regression } & \multicolumn{3}{|c|}{ Multiple regression } \\
\hline & B & SE & $\mathrm{p}$ & $B$ & SE & $\mathrm{p}$ & B & SE & $\mathrm{p}$ & B & SE & $\mathrm{p}$ & B & $\mathrm{SE}$ & $\mathrm{p}$ & $B$ & $\mathrm{SE}$ & $\mathrm{p}$ \\
\hline HbAlc & 0.019 & 0.005 & $<0.001$ & 0.014 & 0.004 & $<0.001$ & 0.010 & 0.002 & $<0.001$ & 0.009 & 0.002 & $<0.001$ & 0.003 & 0.001 & $<0.001$ & 0.003 & 0.001 & $<0.001$ \\
\hline Age & -0.003 & 0.001 & $<0.001$ & -0.001 & 0.001 & 0.076 & -0.001 & $<0.001$ & $<0.001$ & -0.001 & $<0.001$ & 0.089 & $<0.001$ & $<0.001$ & $<0.001$ & $<0.001$ & $<0.001$ & 0.122 \\
\hline Male & -0.044 & 0.012 & $<0.001$ & -0.022 & 0.012 & 0.055 & -0.013 & 0.005 & 0.019 & -0.001 & $<0.001$ & 0.087 & -0.004 & 0.002 & 0.018 & -0.003 & 0.002 & 0.076 \\
\hline BMI & 0.007 & 0.002 & $<0.001$ & 0.001 & 0.002 & 0.438 & 0.002 & 0.001 & 0.052 & & & & $<0.001$ & $<0.001$ & 0.049 & $<0.001$ & $<0.001$ & 0.467 \\
\hline Current smoking & 0.018 & 0.013 & 0.158 & & & & 0.002 & 0.006 & 0.754 & & & & 0.001 & 0.002 & 0.787 & & & \\
\hline Hypertension & -0.047 & 0.012 & $<0.001$ & -0.024 & 0.011 & 0.030 & -0.018 & 0.005 & $<0.001$ & -0.015 & 0.006 & 0.009 & -0.005 & 0.001 & $<0.001$ & -0.004 & 0.002 & 0.008 \\
\hline eGFR & 0.001 & $<0.001$ & $<0.001$ & $<0.001$ & $<0.001$ & 0.889 & 0.001 & $<0.001$ & 0.014 & $<0.001$ & $<0.001$ & 0.646 & $<0.001$ & $<0.001$ & 0.014 & $<0.001$ & $<0.001$ & 0.694 \\
\hline Types of statins & 0.001 & 0.007 & 0.958 & & & & 0.003 & 0.003 & 0.318 & & & & 0.001 & 0.001 & 0.321 & & & \\
\hline Intensive statin treatment & 0.116 & 0.017 & $<0.001$ & 0.088 & 0.016 & $<0.001$ & 0.037 & 0.007 & $<0.001$ & 0.050 & 0.008 & $<0.001$ & 0.010 & 0.002 & $<0.001$ & 0.014 & 0.002 & $<0.001$ \\
\hline Statin combined with ezetimibe & 0.322 & 0.013 & $<0.001$ & 0.264 & 0.013 & $<0.001$ & 0.131 & 0.006 & $<0.001$ & 0.143 & 0.007 & $<0.001$ & 0.037 & 0.002 & $<0.001$ & 0.040 & 0.002 & $<0.001$ \\
\hline LDL-C (mean) & 0.317 & 0.008 & $<0.001$ & 0.270 & 0.009 & $<0.001$ & & & & & & & & & & & & \\
\hline
\end{tabular}

$\beta$ indicates regression coefficients; $\mathrm{SE}$, standard error; $\mathrm{SD}$, standard deviation; $\mathrm{CV}$, coefficient of variation; VIM, variability independent of the mean; Other abbreviations, refer to Table 1. Significance was set to $\mathrm{P}<0.05$ and highlighted in bold

Table 3 Results of univariate and multivariate linear regression for the variability of non-HDL-C

\begin{tabular}{|c|c|c|c|c|c|c|c|c|c|c|c|c|c|c|c|c|c|c|}
\hline & \multicolumn{6}{|c|}{ SD } & \multicolumn{6}{|c|}{$\mathrm{CV}$} & \multicolumn{6}{|c|}{ VIM } \\
\hline & \multicolumn{3}{|c|}{ Univariate regression } & \multicolumn{3}{|c|}{ Multiple regression } & \multicolumn{3}{|c|}{ Univariate regression } & \multicolumn{3}{|c|}{ Multiple regression } & \multicolumn{3}{|c|}{ Univariate regression } & \multicolumn{3}{|c|}{ Multiple regression } \\
\hline & B & SE & $\mathrm{p}$ & B & SE & $\mathrm{p}$ & B & SE & $\mathrm{p}$ & $B$ & SE & $\mathrm{p}$ & B & SE & $\mathrm{p}$ & B & SE & $\mathrm{p}$ \\
\hline HbAlc & 0.029 & 0.005 & $<0.001$ & 0.023 & 0.004 & $<0.001$ & 0.009 & 0.002 & $<0.001$ & 0.009 & 0.002 & $<0.001$ & 0.001 & $<0.001$ & $<0.001$ & 0.001 & $<0.001$ & $<0.001$ \\
\hline Age & -0.004 & 0.001 & $<0.001$ & -0.002 & 0.001 & 0.006 & -0.001 & $<0.001$ & $<0.001$ & -0.001 & $<0.001$ & 0.003 & $<0.001$ & $<0.001$ & $<0.001$ & $<0.001$ & $<0.001$ & 0.003 \\
\hline Gender & -0.042 & 0.013 & 0.001 & -0.013 & 0.013 & 0.314 & -0.003 & 0.004 & 0.416 & & & & -0.001 & 0.001 & 0.307 & & & \\
\hline BMI & 0.010 & 0.002 & $<0.001$ & 0.001 & 0.002 & 0.686 & 0.002 & 0.001 & 0.013 & $<0.001$ & 0.001 & 0.573 & $<0.001$ & $<0.001$ & 0.007 & $<0.001$ & $<0.001$ & 0.464 \\
\hline Current smoking & 0.025 & 0.013 & 0.067 & & & & 0.008 & 0.004 & 0.057 & & & & 0.001 & 0.001 & 0.082 & & & \\
\hline Hypertension & -0.046 & 0.012 & $<0.001$ & -0.025 & 0.013 & 0.045 & -0.015 & 0.04 & $<0.001$ & -0.010 & 0.005 & 0.029 & -0.002 & 0.001 & $<0.001$ & -0.001 & 0.001 & 0.035 \\
\hline eGFR & 0.001 & 0.001 & $<0.001$ & $<0.001$ & $<0.001$ & 0.660 & $<0.001$ & $<0.001$ & $<0.001$ & $<0.001$ & $<0.001$ & 0.661 & $<0.001$ & $<0.001$ & $<0.001$ & $<0.001$ & $<0.001$ & 0.690 \\
\hline Types of statins & 0.015 & 0.007 & 0.035 & 0.026 & 0.009 & 0.005 & 0.006 & 0.002 & 0.011 & 0.008 & 0.003 & 0.016 & 0.001 & $<0.001$ & 0.017 & 0.001 & $<0.001$ & 0.014 \\
\hline Intensive statin treatment & 0.032 & 0.017 & 0.068 & & & & 0.007 & 0.006 & 0.199 & & & & 0.001 & 0.001 & 0.356 & & & \\
\hline Statin combined with ezetimibe & 0.232 & 0.015 & $<0.001$ & 0.167 & 0.014 & $<0.001$ & 0.069 & 0.005 & $<0.001$ & 0.069 & 0.005 & $<0.001$ & 0.009 & 0.001 & $<0.001$ & 0.009 & 0.001 & $<0.001$ \\
\hline non-HDL-C (mean) & 0.264 & 0.007 & $<0.001$ & 0.247 & 0.008 & $<0.001$ & & & & & & & & & & & & \\
\hline
\end{tabular}

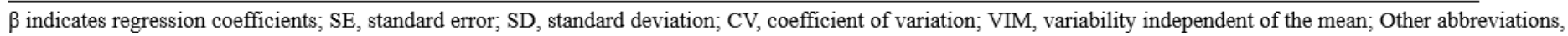
refer to Table 1. Significance was set to $P<0.05$ and highlighted in bold 
Table 4 Results of univariate and multivariate linear regression for the variability of TC

\begin{tabular}{|c|c|c|c|c|c|c|c|c|c|c|c|c|c|c|c|c|c|c|}
\hline & \multicolumn{6}{|c|}{ SD } & \multicolumn{6}{|c|}{$\mathrm{CV}$} & \multicolumn{6}{|c|}{ VIM } \\
\hline & \multicolumn{3}{|c|}{ Univariate regression } & \multicolumn{3}{|c|}{ Multiple regression } & \multicolumn{3}{|c|}{ Univariate regression } & \multicolumn{3}{|c|}{ Multiple regregsion } & \multicolumn{3}{|c|}{ Univariate regreasion } & \multicolumn{3}{|c|}{ Multiple regression } \\
\hline & B & SE & $\mathrm{p}$ & $B$ & SE & $\mathrm{p}$ & $B$ & SE & $\mathrm{p}$ & $B$ & $\mathrm{SE}$ & p & $B$ & $\mathrm{SE}$ & $\mathrm{p}$ & $B$ & SE & $\mathrm{p}$ \\
\hline HbAlc & 0.048 & 0.006 & $<0.001$ & 0.041 & 0.005 & $<0.001$ & 0.012 & 0.001 & $<0.001$ & 0.011 & 0.001 & $<0.001$ & 0.001 & $<0.001$ & $<0.001$ & 0.001 & $<0.001$ & $<0.001$ \\
\hline Age & -0.005 & 0.001 & $<0.001$ & -0.003 & 0.001 & 0.001 & -0.001 & $<0.001$ & $<0.001$ & -0.001 & $<0.001$ & 0.001 & $<0.001$ & $<0.001$ & $<0.001$ & $<0.001$ & $<0.001$ & 0.001 \\
\hline Gender & -0.071 & 0.016 & $<0.001$ & -0.001 & 0.015 & 0.930 & -0.005 & 0.004 & 0.153 & & & & $<0.001$ & $<0.001$ & 0.159 & & & \\
\hline BMI & 0.011 & 0.002 & $<0.001$ & 0.006 & 0.002 & 0.006 & 0.002 & 0.001 & $<0.001$ & 0.002 & 0.001 & 0.007 & $<0.001$ & $<0.001$ & $<0.001$ & $<0.001$ & $<0.001$ & 0.001 \\
\hline Current smoking & 0.012 & 0.016 & 0.438 & & & & 0.005 & 0.004 & 0.151 & & & & $<0.001$ & $<0.001$ & 0.322 & & & \\
\hline Hypertension & -0.036 & 0.015 & 0.014 & -0.007 & 0.015 & 0.642 & -0.006 & 0.003 & 0.073 & & & & $<0.001$ & $<0.001$ & 0.134 & & & \\
\hline eGFR & 0.002 & 0.001 & $<0.001$ & $<0.001$ & $<0.001$ & 0.923 & $<0.001$ & $<0.001$ & 0.001 & $<0.001$ & $<0.001$ & 0.956 & $<0.001$ & $<0.001$ & $<0.001$ & $<0.001$ & $<0.001$ & 0.880 \\
\hline Types of statins & 0.004 & 0.009 & 0.672 & & & & 0.001 & 0.002 & 0.456 & & & & $<0.001$ & $<0.001$ & 0.410 & & & \\
\hline Intensive statin treatment & 0.104 & 0.021 & $<0.001$ & 0.306 & 0.017 & $<0.001$ & 0.024 & 0.005 & $<0.001$ & 0.031 & 0.005 & $<0.001$ & 0.002 & $<0.001$ & $<0.001$ & 0.002 & $<0.001$ & $<0.001$ \\
\hline Statin combined with ezetimibe & 0.379 & 0.017 & $<0.001$ & 0.240 & 0.009 & $<0.001$ & 0.080 & 0.004 & $<0.001$ & 0.085 & 0.004 & $<0.001$ & 0.006 & $<0.001$ & $<0.001$ & 0.006 & $<0.001$ & $<0.001$ \\
\hline TC (mean) & 0.279 & 0.008 & $<0.001$ & 0.240 & 0.009 & $<0.001$ & & & & & & & & & & & & \\
\hline
\end{tabular}

$\beta$ indicates regression coefficients; SE, standard error; SD, standard deviation; CV, coefficient of variation; VIM, variability independent of the mean; Other abbreviations, refer to Table 1. Significance was set to $P<0.05$ and highlighted in bold

Table 5 Results of univariate and multivariate linear regression for the variability of TG

\begin{tabular}{|c|c|c|c|c|c|c|c|c|c|c|c|c|c|c|c|c|c|c|}
\hline & \multicolumn{6}{|c|}{ SD } & \multicolumn{6}{|c|}{$\mathrm{CV}$} & \multicolumn{6}{|c|}{ VIM } \\
\hline & \multicolumn{3}{|c|}{ Univariate regression } & \multicolumn{3}{|c|}{ Multiple regression } & \multicolumn{3}{|c|}{ Univariate regression } & \multicolumn{3}{|c|}{ Multiple regression } & \multicolumn{3}{|c|}{ Univariate regression } & \multicolumn{3}{|c|}{ Multiple regression } \\
\hline & B & $\mathrm{SE}$ & $\mathrm{p}$ & B & SE & p & $B$ & $\mathrm{SE}$ & $\mathrm{p}$ & $B$ & SE & $p$ & $B$ & SE & $\mathrm{p}$ & $B$ & $\mathrm{SE}$ & $\mathrm{p}$ \\
\hline HbAlc & 0.070 & 0.008 & $<0.001$ & 0.033 & 0.005 & $<0.001$ & 0.018 & 0.002 & $<0.001$ & 0.018 & 0.002 & $<0.001$ & 0.004 & $<0.001$ & $<0.001$ & 0.004 & $<0.001$ & $<0.001$ \\
\hline Age & -0.009 & 0.001 & $<0.001$ & 0.001 & 0.001 & 0.378 & -0.002 & $<0.001$ & $<0.001$ & -0.001 & $<0.001$ & $<0.001$ & $<0.001$ & $<0.001$ & $<0.001$ & $<0.001$ & $<0.001$ & $<0.001$ \\
\hline Male & -0.002 & 0.023 & 0.948 & & & & 0.008 & 0.005 & 0.128 & & & & 0.002 & 0.001 & 0.081 & & & \\
\hline BMI & 0.024 & 0.003 & $<0.001$ & -0.008 & 0.002 & $<0.001$ & 0.004 & 0.001 & $<0.001$ & 0.001 & 0.001 & 0.134 & 0.001 & $<0.001$ & $<0.001$ & $<0.001$ & $<0.001$ & 0.103 \\
\hline Current smoking & 0.051 & 0.024 & 0.034 & 0.001 & 0.016 & 0.967 & 0.014 & 0.006 & 0.013 & 0.003 & 0.007 & 0.613 & 0.002 & 0.001 & 0.023 & 0.001 & 0.001 & 0.533 \\
\hline Hypertension & -0.002 & 0.022 & 0.932 & & & & -0.008 & 0.005 & 0.127 & & & & -0.001 & 0.001 & 0.317 & & & \\
\hline eGFR & 0.002 & $<0.001$ & $<0.001$ & 0.001 & $<0.001$ & 0.001 & $<0.001$ & $<0.001$ & $<0.001$ & $<0.001$ & $<0.001$ & 0.901 & $<0.001$ & $<0.001$ & $<0.001$ & $<0.001$ & $<0.001$ & 0.498 \\
\hline Types of statins & 0.009 & 0.013 & 0.503 & & & & -0.002 & 0.003 & 0.489 & & & & $<0.001$ & 0.001 & 0.619 & & & \\
\hline Intensive statin treatment & 0.015 & 0.032 & 0.641 & & & & 0.020 & 0.007 & 0.007 & 0.017 & 0.009 & 0.055 & 0.003 & 0.001 & 0.013 & 0.003 & 0.002 & 0.070 \\
\hline Statin combined with ezetimibe & 0.083 & 0.027 & 0.002 & 0.031 & 0.017 & 0.066 & 0.022 & 0.006 & $<0.001$ & 0.019 & 0.007 & 0.007 & 0.004 & 0.001 & 0.001 & 0.003 & 0.001 & 0.040 \\
\hline TG (mean) & 0.622 & 0.008 & $<0.001$ & 0.560 & 0.008 & $<0.001$ & & & & & & & & & & & & \\
\hline
\end{tabular}

$\beta$ indicates regression coefficients; $\mathrm{SE}$, standard error; $\mathrm{SD}$, standard deviation; $\mathrm{CV}$, coefficient of variation; VIM, variability independent of the mean; Other abbreviations, refer to Table 1 . Significance was set to $P<0.05$ and highlighted in bold

\section{Results of the multivariate linear regression in subgroups}

In multivariate subgroup analyses, the relationship between $\mathrm{HbA} 1 \mathrm{c}$ and the variability of LDL-C, non-HDLC, TC, and TG did not significantly vary across several subgroups such as non-diabetes, diabetes, atorvastatin, rosuvastatin, regular statin, intensive statin and statin-ezetimibe combined therapy.

Figures 1-3 present the results of subgroup analyses when lipid variability was represented by SD. Similarly, when CV and VIM were employed to represent lipid variability, respectively, subgroup analysis results were consistent with results when using SD. (see Table 6). 
Table 6 Results of multivariate linear regression for the effect of HbAlc on variability in subgroups

\begin{tabular}{|c|c|c|c|c|c|c|c|c|c|c|c|c|c|c|c|c|c|c|c|c|c|}
\hline & \multicolumn{3}{|c|}{ non-DM $(n=3306)$} & \multicolumn{3}{|c|}{$\mathrm{DM}(\mathrm{n}=1139)$} & \multicolumn{3}{|c|}{ Atorvastatin $(n=2687)$} & \multicolumn{3}{|c|}{ Rosuvastatin ( $n=1559$ ) } & \multicolumn{3}{|c|}{$\operatorname{RS}(\mathrm{n}=2681)$} & \multicolumn{3}{|c|}{ IS $(n=764)$} & \multicolumn{3}{|c|}{$R S+E \quad(n=875)$} \\
\hline & $\beta$ & $95 \% \mathrm{Cl}$ & p-ralue & $\beta$ & $95 \% \mathrm{Cl}$ & $p$-ralue & $\beta$ & $95 \% \mathrm{Cl}$ & p-ralue & $\beta$ & $95 \% \mathrm{Cl}$ & $p$-ralue & $\beta$ & $95 \% \mathrm{Cl}$ & p-value & $\beta$ & $95 \% \mathrm{Cl}$ & $p$-ralue & $\beta$ & $95 \% \mathrm{Cl}$ & p-value \\
\hline LDL-C (CV) & 0.008 & $0.000-0.017$ & 0.002 & 0.010 & $0.004-0.017$ & 0.003 & 0.009 & $0.004-0.014$ & $<0.001$ & 0.011 & $0.004-0.019$ & 0.002 & 0.010 & $0.005-0.014$ & $<0.001$ & 0.012 & $0.002-0.022$ & 0.019 & 0.012 & $0.003-0.021$ & 0.015 \\
\hline LDL-C (VIM) & 0.002 & $0.000-0.005$ & 0.039 & 0.003 & $0.001-0.005$ & 0.003 & 0.002 & $0.001-0.004$ & $<0.001$ & 0.003 & $0.001-0.005$ & 0.002 & 0.003 & $0.001-0.004$ & $<0.001$ & 0.004 & $0.001-0.007$ & 0.014 & 0.003 & $0.000-0.007$ & 0.023 \\
\hline non-HDL-C (CV) & 0.014 & $0.007-0.021$ & $<0.001$ & 0.009 & $0.004-0.014$ & 0.001 & 0.006 & $0.002-0.010$ & 0.001 & 0.014 & $0.008-0.020$ & $<0.001$ & 0.009 & $0.005-0.012$ & $<0.001$ & 0.008 & $0.002-0.014$ & 0.012 & 0.012 & $0.003-0.020$ & $<0.001$ \\
\hline non-HDL-C (VMM) & 0.002 & $0.001-0.003$ & $<0.001$ & 0.001 & $0.000-0.002$ & 0.001 & 0.001 & $0.000-0.001$ & 0.002 & 0.002 & $0.001-0.003$ & $<0.001$ & 0.001 & $0.001-0.002$ & $<0.001$ & 0.001 & $0.000-0.002$ & 0.009 & 0.002 & $0.001-0.003$ & $<0.001$ \\
\hline $\mathrm{TC}(\mathrm{CV})$ & 0.017 & $0.012-0.022$ & $<0.001$ & 0.011 & $0.007-0.015$ & $<0.001$ & 0.009 & $0.006-0.012$ & $<0.001$ & 0.016 & $0.012-0.021$ & $<0.001$ & 0.01 & $0.007-0.013$ & $<0.001$ & 0.015 & $0.006-0.024$ & $<0.001$ & 0.015 & $0.008-0.023$ & $<0.001$ \\
\hline $\mathrm{TC}(\mathrm{VTM})$ & 0.001 & $0.001-0.002$ & $<0.001$ & 0.001 & $0.000-0.001$ & $<0.001$ & 0.001 & $0.000-0.001$ & $<0.001$ & 0.001 & $0.001-0.002$ & $<0.001$ & 0.001 & $0.000-0.001$ & $<0.001$ & 0.001 & $0.000-0.002$ & $<0.001$ & 0.001 & $0.001-0.002$ & $<0.001$ \\
\hline $\mathrm{TG}(\mathrm{CV})$ & 0.031 & $0.023-0.040$ & $<0.001$ & 0.013 & $0.006-0.021$ & 0.001 & 0.014 & $0.009-0.020$ & $<0.001$ & 0.026 & $0.018-0.033$ & $<0.001$ & 0.015 & $0.009-0.020$ & $<0.001$ & 0.033 & $0.017-0.049$ & $<0.001$ & 0.02 & $0.012-0.033$ & $<0.001$ \\
\hline TG (VIM) & 0.006 & $0.004-0.008$ & $<0.001$ & 0.003 & $0.001-0.005$ & $<0.001$ & 0.003 & $0.002-0.004$ & $<0.001$ & 0.006 & $0.004-0.007$ & $<0.001$ & 0.003 & $0.002-0.004$ & $<0.001$ & 0.008 & $0.004-0.011$ & $<0.001$ & 0.005 & $0.003-0.007$ & $<0.001$ \\
\hline
\end{tabular}

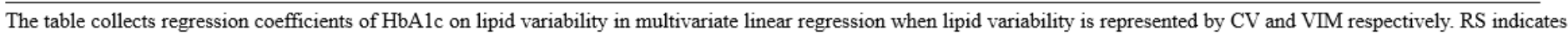

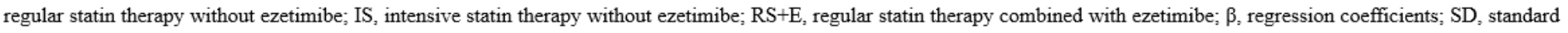
deviation; $\mathrm{CI}$, confidence interval; $\mathrm{CV}$, coefficient of variation; VIM, variability independent of the mean. Other abbreviations, refer to Table 1 .

\section{Discussion}

The main findings of this study are summarized as follows: (1) HbA1c level was a potential risk factor for the variability of LDL-C, non-HDL-C, TC and TG. The results were consistent when SD, CV, and VIM were used to represent lipid variability, respectively; (2) Subgroup analysis demonstrated that the effect HbA1c on the variability of LDL-C, non-HDL-C, TC, and TG remained similar in several relevant subgroups. In patients without diabetes, the significant positive correlations between $\mathrm{HbA} 1 \mathrm{c}$ and lipids variability remained, including LDL-C, non-HDL-C, TC and TG.

Elevated visit-to-visit variability of blood lipids is associated with different adverse outcomes or organ dysfunction, including CVD, obstructive sleep apnea, renal function decline and cognitive decline[18-20]. Based on the Treating to New Targets (TNT) trial, the visit-to-visit variability of LDL-C, HDL-C and TG was demonstrated to be an independent predictor of cardiovascular events[21, 22]. Moreover, Lee et al.[12] confirmed that the visit-to-visit variability of non-HDL-C was associated with major adverse cardiovascular and cerebrovascular events (MACCE) in patients who had undergone PCl. Furthermore, a study based on 3.6 million people in the general population showed that the variability of TC was associated with the risk of all-cause mortality, $\mathrm{Ml}$ and stroke[23]. A wealth of data suggests that the visitto-visit variability of lipid profiles is independently associated with the risk of CVD. However, the underlying mechanisms for the association are still unclear. Similarly, $\mathrm{HbA} 1 \mathrm{c}$ has been identified as a risk factor for CVD and confirmed by many epidemiological studies $[4,5,24,25]$. The current study has demonstrated the potential role of $\mathrm{HbA} 1 \mathrm{c}$ as a risk factor for the variability of $\mathrm{LDL}-\mathrm{C}$, non-HDL-C, TC, and TG. To further explore the relationship between $\mathrm{HbA1c}$ and blood lipid variability, we postulate the following underlying mechanisms based on findings from previous studies.

Insulin resistance $(\mathrm{IR})$ is considered one of the underlying mechanisms, which play an essential role in glucose and lipid metabolism. In this study, IR may be ubiquitous among subjects. A quarter of the patients had a history of type $2 \mathrm{DM}$, which is very likely to be accompanied by $\operatorname{IR}[26]$. Besides, within the normal range of glucose tolerance, patients with CVD have more IR compared to those without CVD, which suggests that patients without type 2 DM in this study may also have IR[27]. IR not only affects $\mathrm{HbA1c}$ levels by reducing glucose transport into cells and glycogen synthesis, but is also closely related 
to dyslipidemia[28]. Although the existing evidence is not sufficient to ultimately demonstrate the effect of IR on lipid variability, the evidence for the role of IR in lipid metabolism appears to suggest this possibility. IR leads to dyslipidemia via the following ways: (1) increased TG, (2) decreased HDL-C, and (3) the appearance of small dense LDL particles[29]. Moreover, hyperinsulinemia, which is the response of insulin resistance, stimulates the synthesis and secretion of very LDL and promotes LDL-C transport into arterial smooth muscle cells[30,31]. In general, given the high prevalence of IR in our patients and its effects on glucose and lipid metabolism, IR could be the potential mechanism behind our findings[32].

Based on analyses of baseline characteristics, the variability of LDL-C, non-HDL-C, TC and TG showed significant differences between categories of $\mathrm{HbA} 1 \mathrm{c}$. However, this finding was not observed for the variability of HDL-C. Unlike other blood lipids, HDL-C is often called "good cholesterol," which exerts multiple beneficial functions within the cardiovascular system[33]. There is a negative correlation between $\mathrm{HbA} 1 \mathrm{c}$ and HDL-C levels. Reduced HDL-C levels often accompany elevated HbA1c[34]. To some extent, it is possible that this negative correlation weakened the influence of $\mathrm{HbA} 1 \mathrm{c}$ on the variability of HDL-C, hence the null findings.

Both $\mathrm{HbA1c}$ and blood lipid variability are risk factors for adverse CVD outcomes. Based on the data of patients undergoing elective $\mathrm{PCl}$, this study clarified the relationship between $\mathrm{HbA} 1 \mathrm{c}$ and blood lipid variability. First, insights on the relationships promote the understanding of the role of $\mathrm{HbA} 1 \mathrm{c}$ and lipid variability in CVD development. Second, these findings provide new perspectives for further understanding the relationship between glucose metabolism and lipid metabolism, which are closely related in vivo.

\section{Limitations}

There are a number of limitations to consider. First, being a single-center retrospective study, it is limited by inherent biases. Second, we had no data on antidiabetic medications, which may affect lipid metabolism or improve insulin sensitivity. Third, this study examined the effect of long-term blood glucose levels on lipid variability. Indicators of short-term blood glucose levels or glucose tolerance such as random glucose, fasting plasma glucose and $2 \mathrm{~h}$ post-OGTT glucose, were not included in this study,

\section{Conclusion}

$\mathrm{HbA} 1 \mathrm{c}$ is a potential risk factor for the variability of LDL-C, non-HDLC, TC, and TG in patients undergoing elective PCl.

\section{Abbreviations}

HbA1c: hemoglobin A1c; CVD: cardiovascular disease; PCl: percutaneous coronary intervention; LDL-C: low-density lipoprotein cholesterol; non-HDL-C: non-high-density lipoprotein cholesterol; TC: total cholesterol; TG: and triglyceride; SD: standard deviation; CV: coefficient of variation; VIM: variability 
independent of the mean; DM: diabetes mellitus; MI: myocardial infarction; EF: ejection fraction; BMI: Body mass index; eGFR: estimated Glomerular filtration rate; ACEl: angiotensin-converting enzyme inhibitors; ARB: angiotensin receptor blocker; CCB: calcium channel blocker.

\section{Declarations}

\section{Acknowledgements}

The authors would like to express their gratitude to EditSprings (https://www.editsprings.com/) for the expert linguistic services provided.

\section{Authors' contributions}

WB $Z$ and $Z Y C$ conceived and designed the study. DB L organized these data and drafted the manuscript with the help of C Y, LD Z and Yi L. DB L analysed the data. Ya $L$ drawed the pictures. WB Z, ZY C and GS $F$ detected any errors in the whole process. All authors have read and approved the manuscript for submission.

\section{Funding}

This work was supported by grants from the National Natural Science Foundation of China (82070408), Zhejiang Natural Science Fund (LY18H020007) and Medical Health Science and Technology Project of Zhejiang Provincial Health Commission (2021RC014).

\section{Availability of data and materials}

The datasets used and/or analyzed during the current study are available from the corresponding author on reasonable request.

\section{Ethics approval and consent to participate}

The study was given approval by the Ethics Committee of Sir Run Run Shaw Hospital of Zhejiang University.

\section{Consent for publication}

Not applicable.

\section{Competing interests}

The authors declare that they have no competing interests.

\section{References}


1. Joseph P, Leong D, McKee M, Anand SS, Schwalm J-D, Teo K, Mente A, Yusuf S: Reducing the Global Burden of Cardiovascular Disease, Part 1: The Epidemiology and Risk Factors.Circulation research 2017, 121:677-694.

2. Chatterjee S, Khunti K, Davies MJ: Type 2 diabetes.Lancet (London, England) 2017, 389:2239-2251.

3. American Diabetes A: 6. Glycemic Targets: Standards of Medical Care in Diabetes-2020.Diabetes Care 2020, 43:S66-S76.

4. Wan EYF, Yu EYT, Chen JY, Wong ICK, Chan EWY, Lam CLK: Associations between usual glycated haemoglobin A1c and Cardiovascular Disease in Patients with Type 2 Diabetes Mellitus: A 10-year Diabetes cohort study.Diabetes Obes Metab 2020:1-10.

5. Zhang H, Qin L, Sheng CS, Niu Y, Gu H, Lu S, Yang Z, Tian J, Su Q: ASCVD risk stratification modifies the effect of $\mathrm{HbA1c}$ on cardiovascular events among patients with type 2 diabetes mellitus with basic to moderate risk.BMJ Open Diabetes Res Care 2020, 8.

6. Gerstein HC: Dysglycemia and cardiovascular risk in the general population.Circulation 2009, 119:773-775.

7. Katzmann JL, Laufs U: New Insights in the Control of Low-Density Lipoprotein Cholesterol to Prevent Cardiovascular Disease.Curr Cardiol Rep 2019, 21:69.

8. Gordon DJ, Probstfield JL, Garrison RJ, Neaton JD, Castelli WP, Knoke JD, Jacobs DR, Jr., Bangdiwala S, Tyroler HA: High-density lipoprotein cholesterol and cardiovascular disease. Four prospective American studies.Circulation 1989, 79:8-15.

9. Boey E, Gay GM, Poh KK, Yeo TC, Tan HC, Lee CH: Visit-to-visit variability in LDL-and HDL-cholesterol is associated with adverse events after ST-segment elevation myocardial infarction: A 5-year followup study.Atherosclerosis 2016, 244:86-92.

10. Han BH, Han K, Yoon KH, Kim MK, Lee SH: Impact of Mean and Variability of High-Density Lipoprotein-Cholesterol on the Risk of Myocardial Infarction, Stroke, and Mortality in the General Population.J Am Heart Assoc 2020, 9:e015493.

11. Wan EYF, Yu EYT, Chin WY, Barrett JK, Mok AHY, Lau CST, Wang Y, Wong ICK, Chan EWY, Lam CLK: Greater variability in lipid measurements associated with cardiovascular disease and mortality: A 10year diabetes cohort study.Diabetes Obes Metab 2020, 22:1777-1788.

12. Lee EY, Yang Y, Kim HS, Cho JH, Yoon KH, Chung WS, Lee SH, Chang K: Effect of visit-to-visit LDL-, HDL-, and non-HDL-cholesterol variability on mortality and cardiovascular outcomes after percutaneous coronary intervention.Atherosclerosis 2018, 279:1-9.

13. Zhao L, Xu T, Li Y, Luan Y, Lv Q, Fu G, Zhang W: Variability in blood lipids affects the neutrophil to lymphocyte ratio in patients undergoing elective percutaneous coronary intervention: a retrospective study.Lipids Health Dis 2020, 19:124.

14. Clark D, 3rd, Nicholls SJ, St John J, Elshazly MB, Kapadia SR, Tuzcu EM, Nissen SE, Puri R: Visit-tovisit cholesterol variability correlates with coronary atheroma progression and clinical outcomes.Eur Heart J 2018, 39:2551-2558. 
15. Levine GN, Bates ER, Blankenship JC, Bailey SR, Bittl JA, Cercek B, Chambers CE, Ellis SG, Guyton RA, Hollenberg SM, et al: 2011 ACCF/AHA/SCAI Guideline for Percutaneous Coronary Intervention: executive summary: a report of the American College of Cardiology Foundation/American Heart Association Task Force on Practice Guidelines and the Society for Cardiovascular Angiography and Interventions. Catheter Cardiovasc Interv 2012, 79:453-495.

16. Rothwell PM, Howard SC, Dolan E, O'Brien E, Dobson JE, Dahlöf B, Sever PS, Poulter NR: Prognostic significance of visit-to-visit variability, maximum systolic blood pressure, and episodic hypertension. The Lancet 2010, 375:895-905.

17. Matsuo S, Imai E, Horio M, Yasuda Y, Tomita K, Nitta K, Yamagata K, Tomino Y, Yokoyama H, Hishida A: Revised equations for estimated GFR from serum creatinine in Japan.American journal of kidney diseases : the official journal of the National Kidney Foundation 2009, 53:982-992.

18. Smit RA, Trompet S, Sabayan B, le Cessie S, van der Grond J, van Buchem MA, de Craen AJ, Jukema JW: Higher Visit-to-Visit Low-Density Lipoprotein Cholesterol Variability Is Associated With Lower Cognitive Performance, Lower Cerebral Blood Flow, and Greater White Matter Hyperintensity Load in Older Subjects.Circulation 2016, 134:212-221.

19. $\mathrm{Ng} \mathrm{G}$, Boey E, Frampton C, Richards AM, Yeo TC, Lee CH: Obstructive sleep apnea is associated with visit-to-visit variability in low-density lipoprotein-cholesterol in patients with coronary artery disease.Sleep Breath 2017, 21:271-278.

20. Yan Y, Huang Y, Zhou D, Tang S, Feng Y-q: Visit-to-Visit Variability in Total Cholesterol Correlates with the Progression of Renal Function Decline in a Chinese Community-Based Hypertensive Population.Kidney and Blood Pressure Research 2019, 44:727-742.

21. Bangalore S, Breazna A, DeMicco DA, Wun CC, Messerli FH, Committee TNTS, Investigators: Visit-tovisit low-density lipoprotein cholesterol variability and risk of cardiovascular outcomes: insights from the TNT trial.J Am Coll Cardiol 2015, 65:1539-1548.

22. Waters DD, Bangalore S, Fayyad R, DeMicco DA, Laskey R, Melamed S, Barter PJ: Visit-to-visit variability of lipid measurements as predictors of cardiovascular events.J Clin Lipidol 2018, 12:356366.

23. Kim MK, Han K, Kim HS, Park YM, Kwon HS, Yoon KH, Lee SH: Cholesterol variability and the risk of mortality, myocardial infarction, and stroke: a nationwide population-based study. Eur Heart J 2017, 38:3560-3566.

24. Saydah S, Tao M, Imperatore G, Gregg E: GHb level and subsequent mortality among adults in the U.S.Diabetes Care 2009, 32:1440-1446.

25. Palta P, Huang ES, Kalyani RR, Golden SH, Yeh HC: Hemoglobin A1c and Mortality in Older Adults With and Without Diabetes: Results From the National Health and Nutrition Examination Surveys (1988-2011).Diabetes Care 2017, 40:453-460.

26. Shulman GI: Cellular mechanisms of insulin resistance. The Journal of Clinical Investigation 2000, 106:171-176. 
27. Reaven G: Insulin resistance and coronary heart disease in nondiabetic individuals. Arterioscler Thromb Vasc Biol 2012, 32:1754-1759.

28. Yaribeygi $H$, Farrokhi FR, Butler AE, Sahebkar A: Insulin resistance: Review of the underlying molecular mechanisms. Journal of Cellular Physiology 2018, 234:8152-8161.

29. Ormazabal V, Nair S, Elfeky O, Aguayo C, Salomon C, Zuniga FA: Association between insulin resistance and the development of cardiovascular disease.Cardiovasc Diabeto/ 2018, 17:122.

30. Porter Karen E, Riches K: The vascular smooth muscle cell: a therapeutic target in Type 2 diabetes? Clinical Science 2013, 125:167-182.

31. Scherer T, Lindtner C, O'Hare J, Hackl M, Zielinski E, Freudenthaler A, Baumgartner-Parzer S, Tödter K, Heeren J, Krššák M, et al: Insulin Regulates Hepatic Triglyceride Secretion and Lipid Content via Signaling in the Brain.Diabetes 2016, 65:1511-1520.

32. P R Shepherd BBK: Glucose transporters and insulin action-implications for insulin resistance and diabetes mellitus. The New England Journal of Medicine 1999, 341:248-257.

33. Toth PP: Cardiology patient page. The "good cholesterol": high-density lipoprotein.Circulation 2005, 111:e89-e91.

34. Pacilli A, De Cosmo S, Trischitta V, Bacci S: Role of relationship between HbA1c, fibrinogen and HDLcholesterol on cardiovascular disease in patients with type 2 diabetes mellitus. Atherosclerosis 2013, 228:247-248.

\section{Figures}

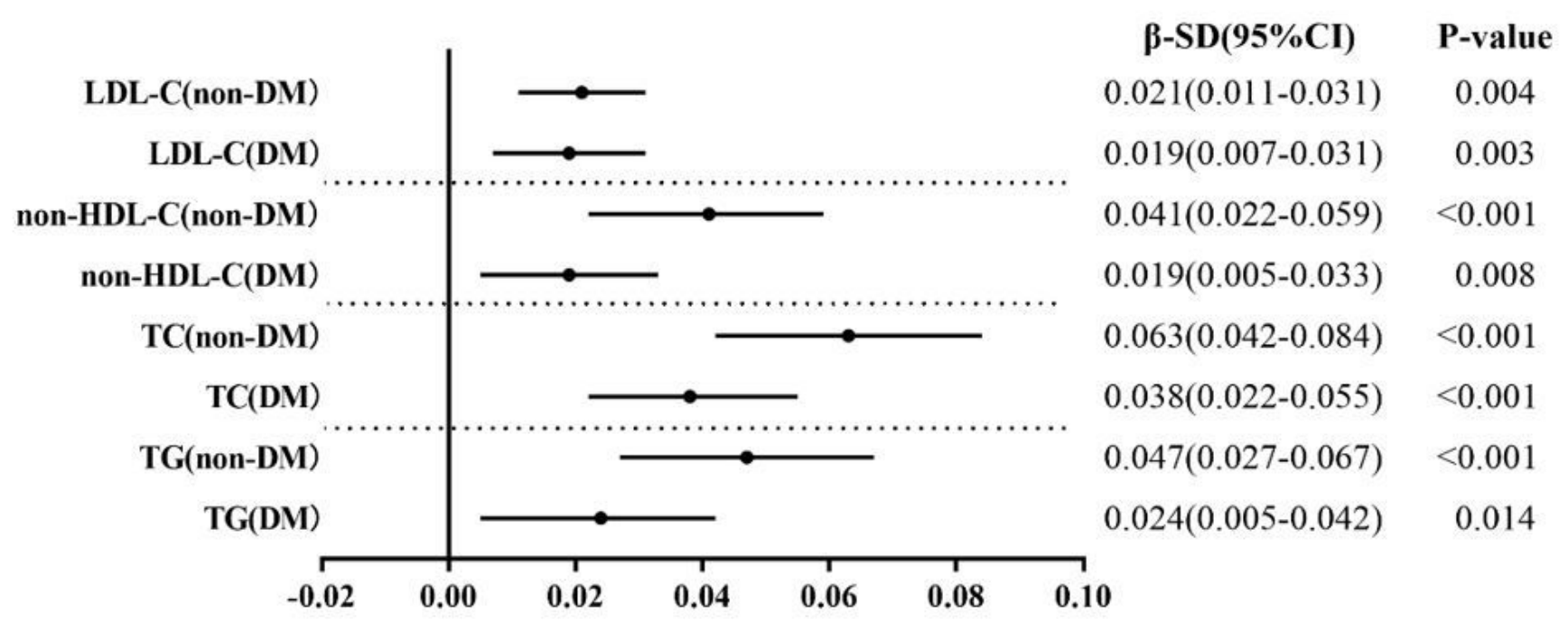

Figure 1

Multivariate analysis for the effect of HbA1c on lipid variability in patients with and without diabetes. Multivariate linear regression results for the effect of $\mathrm{HbA} 1 \mathrm{c}$ on lipid variability, which was represented by 
SD. non-DM indicates group without diabetes; DM, patients with diabetes; $\beta$-SD, regression coefficients of $\mathrm{HbA} 1 \mathrm{c}$ on SD; $\mathrm{Cl}$, confidence intervals.

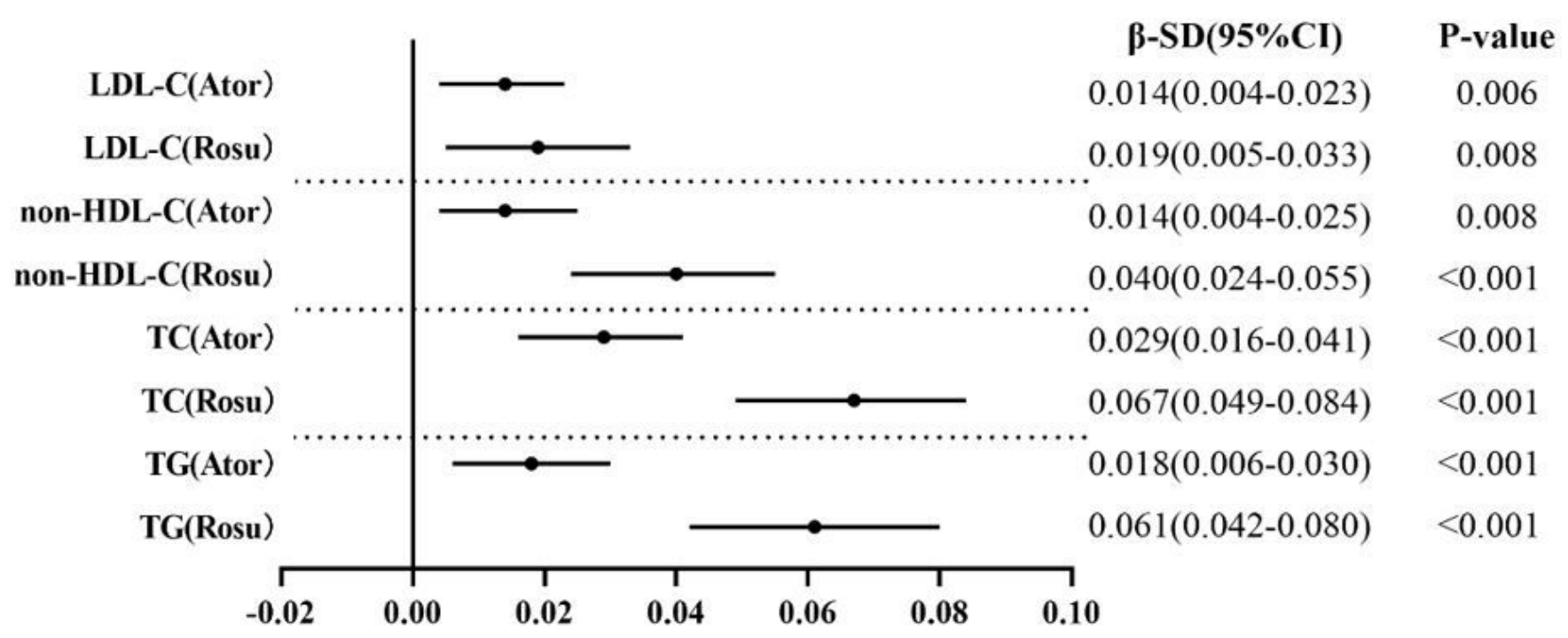

Figure 2

Multivariate analysis for the effect of $\mathrm{HbA} 1 \mathrm{c}$ on lipid variability in Atorvastatin and Rosuvastatin therapy groups. Multivariate linear regression results for the effect of $\mathrm{HbA} 1 \mathrm{c}$ on lipid variability, which was represented by SD. Ator indicates atorvastatin therapy group; Rosu, rosuvastatin therapy group; $\beta$-SD, regression coefficients of $\mathrm{HbA} 1 \mathrm{c}$ on $\mathrm{SD} ; \mathrm{Cl}$, confidence intervals.

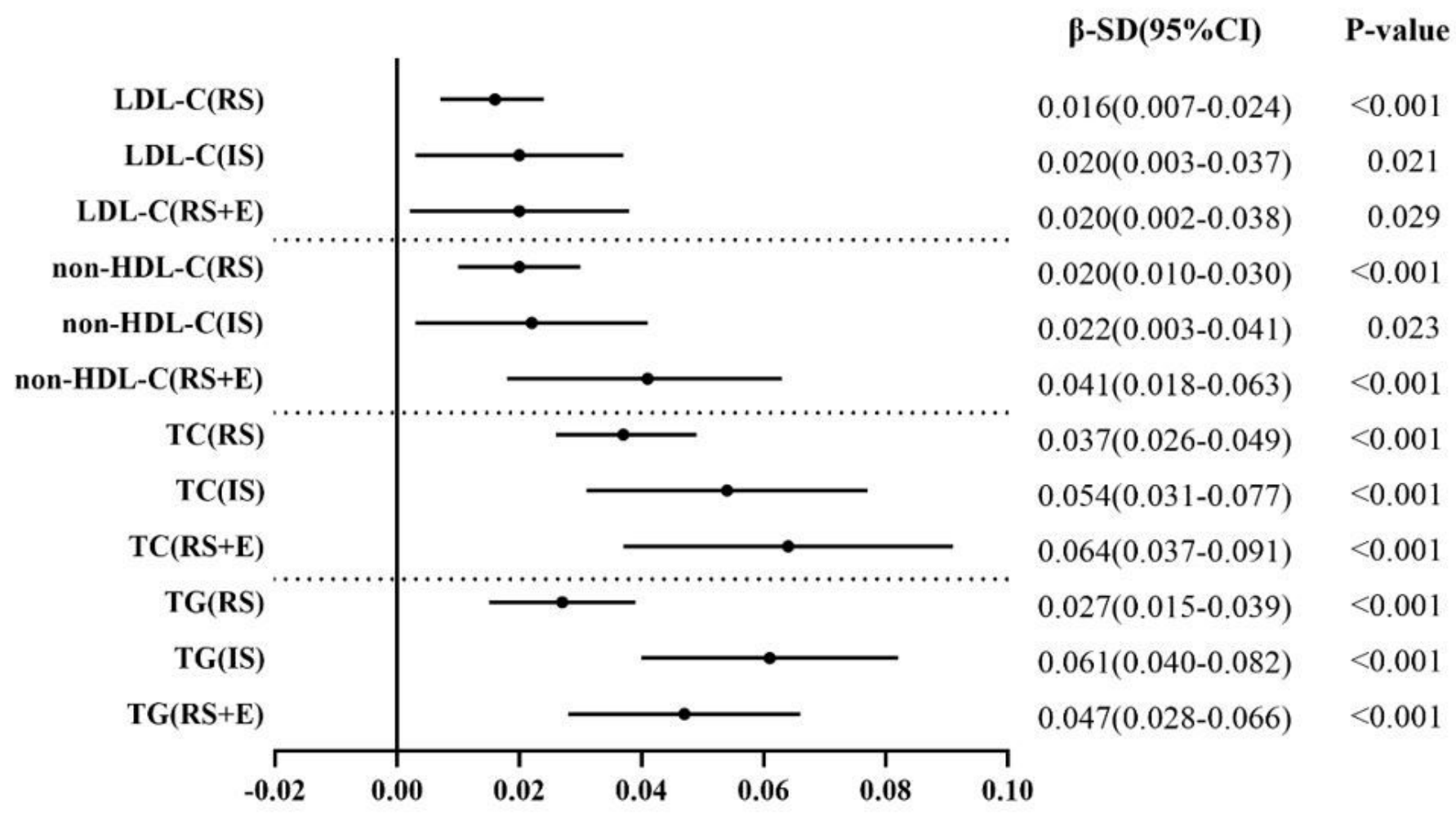




\section{Figure 3}

Multivariate analysis for the effect of $\mathrm{HbA} 1 \mathrm{c}$ on lipid variability in the regular statin, intensive statin, and statin-ezetimibe combined therapy groups. Multivariate linear regression results for the effect of $\mathrm{HbA} 1 \mathrm{c}$ on lipid variability, which was represented by SD. RS indicates regular statin therapy without ezetimibe; IS, intensive statin therapy without ezetimibe; RS+E, regular statin therapy combined with ezetimibe; $\beta-S D$, regression coefficients of $\mathrm{HbA} 1 \mathrm{c}$ on $\mathrm{SD} ; \mathrm{Cl}$, confidence intervals. 